\title{
Recepción del conductismo en Argentina y Brasil: Un estudio de dos universidades, 1960-1970*
}

\author{
Behaviorism reception in Argentina and Brazil: \\ A study of two universities, 1960-1970
}

\author{
FERnANDO POLANCO ** \\ Universidad Nacional de San Luis/CONICET, \\ San Luis, Argentina; \\ RODRIGO LOPES MIRANDA \\ Universidade Federal de Minas Gerais, Brasil.
}

doi:10.11144/Javeriana.upsy13-5.rcab

Para citar este artículo: Polanco, F., \& Lopes, R. (2014). Recepción del conductismo en Argentina y Brasil: un estudio comparativo (1960-1970). Universitas Psychologica, 13(5), 2035-2045. http://dx.doi. org/10.11144/Javeriana.upsy13-5.rcab

El trabajo desarrollado con fondos del Ministerio de Educación, CAPES, Brasil; y Consejo Nacional de Investigación y Ciencia, CONICET, Argentina. Fernando Polanco, Universidad Nacional de San Luis / CONICET, Argentina. Rodrigo Lopes Miranda, Universidade Federal de Minas Gerais, Brasil.

** La correspondencia debe ser enviada a Fernando Polanco, Universidad Nacional de San Luis. Av. Ejército de los Andes 950 - D5700HHW - San Luis - Argentina. Correo electrónico:fernandoapolanco@ gmail.com

\section{RES U M N}

Describimos y analizamos la recepción del conductismo en Argentina y Brasil, a través de dos ejemplos: la Universidad Nacional de San Luis y la Universidade Federal de Minas Gerais. En Argentina, desde 1960 el conductismo fue ampliamente criticado. Excepcionalmente en San Luis en la década de 1970 hubo un grupo de estudiantes y psicólogos jóvenes que fue receptivo a este modelo por razones ideológicas, profesionales y científica. En Brasil, durante esas décadas, la creación de carreras de grado de psicología comenzó a extenderse. El conductismo circuló a través del laboratorio didáctico del análisis del comportamiento. La recepción del conductismo en estos países nos ayuda a entender la circulación del conocimiento psicológico en diferentes lugares. También nos muestra cómo cada uno de estos sitios ha incorporado el conductismo en su propio contexto.

\section{Palabras Clave}

historia de la psicología; historia del conductismo, historia de la psicología en Argentina, historia de la psicología en Brasil

\section{A B S T R A C T}

We are describing and analyzing the reception of behaviorism in Argentina and Brazil in the 1960s and 1970s. We look deeply at two examples: the Universidad Nacional de San Luis and the Universidade Federal de Minas Gerais. In Argentina, the first psychological courses took place during the late 1950s and early 1960s when behaviorism was widely criticized. In the 1970s, exceptionally in San Luis, there was a group of students and young psychologists that were receptive to its model for ideological, professional and scientific reasons. In Brazil, during those decades, the creation of psychology undergraduate courses started to spread. Behaviorism has circulated through the didactic laboratory of behavior analysis in those psychology courses. The reception of behaviorism in these countries helps us to understand the circulation of psychological knowledge in different places. It also shows us how each one of these places incorporated behaviorism uniquely into its own context.

Keyword

history of psychology; history of behaviorism; history of psychology in Argentina; history of psychology in Brazil 
Recepción del conductismo en Argentina y Brasil: Un estudio de dos universidades, 1960-1970

La historiografía de la ciencia en las últimas décadas, ha incorporado nuevos valores y perspectivas, ampliando los objetivos del análisis historiográfico. Dentro de éstos, encontramos el estudio de las "hibridaciones entre las culturas científicas y los saberes locales" (Obregón, 2000, p. 16).

En el caso particular de la historia de la psicología, Danziger (2006) indica que la psicología puede producir historias que son a la vez globales y locales, destacándose las formas de circulación del conocimiento psicológico. Mostrando por un lado las relaciones del conocimiento psicológico en diferentes países y contextos; y por otro, entendiendo que las psicologías se produjeron de manera diferente por contingencias específicas de cada país. La asociación entre estas dos perspectivas nos permite un contacto con los aspectos culturales, sociales e institucionales que están presentes en la construcción de la psicología en diferentes localidades. Es por ello de la importancia de llevar adelante estudios de la recepción de los diferentes sistemas y/o escuelas psicológicas a través del método de la historia comparada, que consiste en:

...elegir, en uno o más medios sociales diferentes, dos o más fenómenos que a primera vista parecen presentar ciertas analogías entre sí, describir sus curvas evolutivas, constatar las similitudes y las diferencias y explicarlas en la medida de lo posible (Bloch, 1999, p. 115).

Para ello, consideremos comparar los procesos de recepción del conductismo en Brasil y Argentina; países que han sido objeto de la historia comparada desde hace algunos años (e.g., Buchbinder, 2010; Capelato, 1998). Escogemos dos instituciones como casos de estudio: (1) la Universidad Nacional de San Luis (UNSL), en la ciudad de San Luis, Argentina; y (2) la Universidade Federal de Minas Gerais (UFMG), en la ciudad de Belo Horizonte, Brasil. Ambas instituciones tuvieron su recepción en un periodo histórico semejante, entre las décadas de 1960 y 1970, siendo dos universidades del interior de su país e inmersas en los procesos y avatares eco- nómicos y políticos semejantes de la región sudamericana de aquella época. En cuanto al contexto del campo psicológico destaca la conocida diferencia en cuanto a la relevancia que tiene el conductismo en ambos países. Los detalles particulares serán explicitados más adelante.

Para cumplimentar los objetivo del presente estudio se presentaran los siguientes aspectos de cada caso: (a) el contexto general de cada país; (b) un cuadro específico de la enseñanza superior y de la psicología en cada sistema universitario, y (c) como cada recepción del conductismo interactuó con estos aspectos, intentando observar las semejanzas y especificidades de la circulación de los saberes psicológicos alrededor del mundo. Finalmente, esperamos presentar las características singulares de la recepción del conductismo en los casos estudiados. Lo cual nos ayudará a entender la circulación y apropiación de teorías psicológicas en diferentes países (Obregón, 2000; Danziger, 2006).

\section{Ideología, formación y profesión en el conductismo en Argentina}

\section{Modernidad y desarrollo. Ideología y educación en la Argentina del siglo XX}

La modernización en Argentina, se ha expresado de manera heterogénea en diferentes ámbitos sociales. Ha sido objeto de avances y retrocesos constantes debido a crisis socio-históricas, principalmente políticas y económicas, que han generado una inestabilidad y falta de proyecto de desarrollo sostenido a largo plazo. Otro elemento importante en la determinación de la inestabilidad, es la fuerte centralización que el estado argentino ha tenido sobre los diferentes ámbitos de la sociedad, tanto institucionales como culturales.

En el caso de la educación inicial y media, que desde finales de siglo XIX había tenido una fuerte impronta liberal y laica, a mediados de siglo XX sufrió un fuerte cambio a través de la instauración obligatoria de la enseñanza de la religión. Posteriormente fue siendo eliminada, pero siguió formando parte de la educación privada que se 
instauró a partir de la promoción de los sectores conservadores y oligárquicos de la sociedad argentina (Puiggrós, 2003).

La educación superior, desde las reformas de principios de siglo XX, se identificó con una fuerte tendencia a la formación de profesionales liberales. Fue así, que a pesar de que en muchos momentos históricos se intentó promover la formación y promoción de la ciencia dentro de la misma, ésta nunca logro instaurarse dentro de dicho ámbito (Buchbinder, 2010). Por ejemplo, durante el proceso de fuerte industrialización que comenzó en la década de 1940, Hurtado (2010) señala:

...el gobierno peronista se centró en la promoción de áreas vinculadas al desarrollo de la "técnica" y en la profundización del proceso de industrialización. En todo caso, la actividad científica apareció en el discurso oficial como subisidiaria del desarrollo técnico e industrial (p.74)

Fue así, que se fortalecieron formaciones técnicas como las diversas ramas de la ingeniería, que en última instancia no escapaban a las identidades de las profesiones liberales como las tradicionales abogacía o medicina, que seguían siendo las principales elecciones de los estudiantes universitarios.

Durante el gobierno de facto que derrocó al presidente Juan Domingo Perón, se creó el Consejo Nacional de Investigaciones Científicas y Técnicas (CONICET), espacio asociado al desarrollo de una ciencia básica nacional con una fuerte impronta en el desarrollo de las ciencia exactas y naturales, principalmente en el área biomédica. A pesar de que CONICET intento establecer políticas conjuntas de desarrollo de la ciencia con la universidad, estas no tuvieron éxito y la universidad siguió siendo principalmente una institución de formación profesional.

Finalmente, podemos señalar que durante las políticas de inclusión del peronismo, que incluyeron la eliminación de aranceles y exámenes de ingreso a la universidad en la década de 1950, el sector estudiantil alcanzó una masividad que produjo su fortalecimiento y consolidación organizativa e ideológica; y que llegó a su punto culminante a finales de la década de 1960 siendo uno de los principales sectores que luchara por el cambio social. Sin embargo, tras el golpe militar del año 1976, los estudiantes universitarios fueron perseguidos y aniquilados por el terrorismo de estado, siendo un cuarto del total de desaparecidos que se calcula en una cifra aproximada de treinta mil personas.

\section{Origen y orientaciones de la formación del psicólogo Argentino}

Durante los dos primeros periodos de gobierno peronista desenvueltos entre los años 1946 y 1955 , el desarrollo industrial fue muy importante. Con lo cual se intentaría instaurar en las universidades la formación de psicólogos con orientación psicotécnica, para que se convirtieran en estudiosos de las condiciones de los sujetos para desempeñar diferentes tareas y de esa manera orientarlos para aquello en lo que podrían tener mejor ocupación.

Fue así que en el año 1954, con ayuda del estado se reunirían en Tucumán los principales representantes de la psicología con el objetivo de discutir las bases y estructuras de las carreras de psicología (Klappenbach, 2006). Entre los años 1954 y 1962 se crearon las carreras de psicología en las principales universidades nacionales. Sin embargo, la orientación generalista con una formación marcadamente psicotécnica en algunos centros, se vería desarrollada por pocos años ya que el gobierno de facto que derrocó el gobierno peronista, no continuaría promocionando ninguna meta del gobierno previo en su intento por contrarrestar su popularidad y borrar su influencia sociopolítica.

Fue así que para mediados de los años 1960, diferentes espacios del psicoanálisis que se venían gestando en el ámbito privado aprovecharan la falta de orientación y su popularidad social para ingresar en las universidades públicas e imponer una formación del psicólogo como profesional, principalmente para actuar en el ámbito clínico y desde un punto de vista de la teoría psicoanalítica. Además, como señala Plotkin (2003):

La difusión del psicoanálisis en la Argentina en los 60' fue el resultado de una combinación compleja 
de desarrollos sociales, culturales y políticos que generaron la demanda de un sistema de creencias para interpretar la realidad... Por otra parte, logró satisfacer la demanda de grupos sociales que escapaban a su clientela "natural", la clase media en ascenso. (p.142).

En este sentido, un aspecto importante es que las carreras de psicología nacieron en su mayoría con una fuerte impronta de los campos académicos y profesionales de la filosofía y la medicina, con lo cual el psicoanálisis con su carácter predominantemente clínico y con una teoría con un alto nivel hermenéutico podía cumplir con las exigencias de ambos sectores.

Otro elemento a destacar es la militancia estudiantil universitaria que tuvo una fuerte impronta antiimperialista, y por ello todo lo que pudiera ser asociado con Estados Unidos (EE.UU.) y la Unión Soviética (US), era visto como un intento de colonización. Generando un rechazo explícito por parte de la mayoría de los estudiantes de todas aquellas corrientes que tuvieran una orientación reflexológica o conductista, ya que era visto como una imposición colonizadora que impedía dirigir las fuerzas intelectuales a la liberación del pueblo basada en la realidad nacional y latinoamericana.

Sin embargo, no todas las carreras tendrían una orientación únicamente psicoanalítica. Una de las excepciones fue la carrera de psicología que nació en 1958 bajo la égida de la llamada Universidad Nacional de Cuyo, con sede en San Luis, y que con posterioridad al año 1973 se convertiría en la UNSL. Ésta carrera, nació unida al instituto pedagógico y a la carrera de pedagogía por lo que los contenidos provenientes de este campo, entre ellos los de la enseñanza programada y las explicaciones conductistas del aprendizaje, fueron parte de la formación del psicólogo sanluiseño desde sus inicios.

Por otro lado, la distancia con la influencia cultural e ideológica desarrollada en los principales centros urbanos, hizo que los estudiantes de izquierda tuvieran otra lectura conceptual e ideológica de las teorías psicológicas predominantes en la US y EE.UU., ambas de carácter objetivo y con una fuerte base conductual.

\section{Psicología y militancia estudiantil revolucionaria: desde Pavlov a Skinner.}

Desde mediados de los años 1960, un grupo de estudiantes de la carrera de psicología de la UNSL, se comenzó a reunir en grupos de estudio con dos finalidades, una mayor formación filosófica e ideológica y una mayor formación científica y psicológica. Dicho grupo, supo unir sus convicciones ideológicas de izquierda con una psicología de orientación objetivista. Como señala Aldo Birgier:

\footnotetext{
...estábamos en un clima revolucionario, no era solo que queríamos promover la psicología objetiva, esto solo era una parte del contexto general. Nosotros queríamos cambiar la sociedad, y parece una locura pero el clima era ese. Y entre las cosas que había que cambiar una de ellas era la psicología. La defensa de una cuestión científica tenía que ver con una perspectiva materialista, con muchos componentes marxistas, izquierdistas y socialistas. En cierto modo, la reacción contra el psicoanálisis fue una reacción política. (A. Birgier, comunicación personal, 18 de abril de 2006).
}

Es así que la provincia de San Luis no se encontraba exenta de la movilización estudiantil que se observaba en todo el país, aunque al ser una comunidad pequeña y reducida de estudiantes predispuso a una mayor interacción entre las diferentes agrupaciones de izquierda; haciendo que marxistas, leninistas, trotskistas, maoístas, peronistas, anarquistas, se reunieran en un solo grupo con los rasgos generales de los movimientos estudiantiles de izquierda revolucionaria (Rodríguez-Kauth, 1973).

Dicho grupo comenzó a militar activamente por un cambio en la formación del psicólogo teniendo dos actitudes paralelas. Por un lado, tuvieron una actitud negativa y crítica de las psicologías de corte idealistas, particularmente el psicoanálisis; y por otro lado, tuvieron una actitud positiva en defensa de una psicología objetiva.

En cuanto a las críticas sobre el psicoanálisis, los integrantes de dicho grupo identificaban principalmente cinco: el carácter universal de algunos de sus conceptos sin presentar ninguna prueba 
empírica; su subjetivismo; su carácter hegemónico y exclusivista, dentro del ámbito académico en la Argentina; su principio de autoridad, a partir del cual se cometían abusos por parte de algunos profesores y psicoterapeutas; y finalmente, el carácter ideológicamente capitalista de esta teoría psicológica (L. Amante, comunicación personal, 30 de octubre de 2006; A. Birgier, comunicación personal, 18 de abril de 2006; M. Loizo, comunicación personal, 23 de marzo de 2006; L. Marín, comunicación personal, 27 de marzo de 2006; A. Piracés, comunicación personal, 12 de junio de 2006).

En cuanto al apoyo de una psicología objetiva, sus fundamentos se enfocaron primero en el aspecto ideológico, y posteriormente en aspectos pragmáticos y profesionales. Tal como lo señalan sus integrantes, el primer elemento que determinó que adoptaran esta psicología fue la perspectiva materialista, esta se reflejaba en los trabajos de Iván P. Pavlov quien llevaba adelante una investigación objetiva de los fenómenos psicológicos. El primer contacto que dicho grupo de estudiantes tuvo con estas teorías, fue a través de los contenidos de las materias de grado Psicología I y Psicología II, dictadas por Placido Horas, quien era reconocido por su conocimiento enciclopédico que lo llevaba a ofrecer en sus clases un amplio espectro de desarrollos de la psicología del país y el mundo. En el programa de la materia Psicología II del año 1968, cursada por la mayoría de los integrantes de dicho grupo de estudio, encontramos contenidos sobre el origen del conductismo como doctrina objetivista de la psicología, analizando las tesis fundamentales de Watson, su difusión, méritos y limitaciones, y posteriormente su evolución, principalmente el modelo psicológico de Tolman. Por otro lado, planteaba la doctrina reflexológica como modalidad objetivista en psicología, desarrollando los conceptos reflexológicos pavlovianos y la doctrina del materialismo dialéctico.

Es de destacar, que a través de esta materia tuvieron el primer contacto con una serie de libros de importancia para su formación psicológica objetivista con fundamento materialista. Estos estaban escritos por Harry K. Wells, quien fue un reconocido psicólogo y filósofo materialista norteamericano que lucho por una concepción científica de la psicología. Como señala Thénon (1963), prologuista del primer libro de la serie:

La obra de Harry K. Wells... es uno de los aportes más importantes... en la réplica ideológica del materialismo dialéctico contra todas las corrientes del idealismo filosófico que impregna la producción psicológica contemporánea... Por otra parte da término cabal a los intentos que se proponen conciliar el psicoanálisis con el materialismo dialéctico (p. 9).

Este grupo que había comenzado a reunirse a leer libros tanto de índole ideológica como científica, decidió aglutinarse a instancias de Luis Villarreal y Aldo Birgier, bajo la denominación de Estudiantes de Psicología Científica (EPCI) a partir del año 1969. Durante este período que podemos denominar de formación, y que se dio entre los años 1967 y 1973, el grupo logro consolidar su posición tanto en el ámbito académico como político, participando activamente del centro de estudiantes y de espacios curriculares.

En el año 1972 el Dr. Enrique Marianetti los introdujo en la reflexología pavloviana con un seminario titulado Actividad Nerviosa Superior (A. Piracés, comunicación personal, 12 de junio de 2006). A partir del año 1973, debido a la cada vez más grande cantidad de material especializado y a los requerimientos de los estudiantes se comenzaron a dictar dos materias: Psicoanálisis y Reflexología y otras psicologías objetivas.

Para finales del año 1974, la mayoría de sus integrantes estaban recibidos, y participaban de manera ad honorem u ocupando cargos docentes en diferentes cátedras (UNSL, 1975). Con lo cual sus integrantes decidieron formar el Centro de Estudios de Psicología Objetiva: I. P. Pavlov (CEDEPO). En el año 1974 a través de sus gestiones, lograron que se contratara a Victor Rojas, un psicólogo chileno que se encontraba exiliado en Argentina, para que dictara el curso de posgrado Modificación de conducta basada en la teoría del aprendizaje. Posteriormente, en el año 1975, lograrían que se contratara a uno de los referentes más importantes de la psicología conductista latinoamericana, nos referimos al psi- 
cólogo colombiano Rubén Ardila, quien dictaría el curso Terapia del comportamiento (Calabresi \& Polanco, 2008).

Tras tomar estos cursos y adquirir nuevos materiales bibliográficos, los integrantes de CEDEPO señalaban que ya no se podían considerar solo pavlovianos, sino que al incorporar las nuevas teorías del aprendizaje, se consideraban también skinnerianos. Sin embargo, exceptuando algunos pequeños intentos de instaurar estudios experimentales, las actividades de la mayoría de sus integrantes se realizaron en el ámbito profesional. La modificación de la conducta representó para los integrantes de dicho grupo una ventaja invalorable en el aspecto pragmático de la aplicación de conocimientos técnicos de la psicología al campo educativo, criminológico y clínico. Este mismo hecho, se reflejó en la propia formación del psicólogo sanluiseño, que tenía materias de formación conductista con una fuerte orientación a la práctica profesional.

Finalmente, podemos señalar que si bien las actividades de CEDEPO en el ámbito ideológico y académico eran cada vez más amplias y tenían cada vez un mayor nivel de convocatoria en la carrera de psicología de la UNSL, todo esto se vería truncado con la llegada del golpe militar de 1976, donde se cesanteó a los docentes y expulso a los alumnos más relevantes del grupo tomando como elemento fundamental de la medida sus asociaciones ideológica enmarcadas en la Ley de Seguridad Nacional. En las horas subsiguientes, temiendo por sus vidas y por las de sus conocidos, muchos de sus integrantes quemaron todos los documentos y libros que podrían incriminarlos a ellos y a sus compañeros, y posteriormente huyeron al exilio.

\section{Conductismo como}

\section{Análisis Experimental del} Comportamiento en Brasil

\section{Big Picture: Educación y modernización}

La primera mitad del siglo XX en Brasil fue marcada por el esfuerzo de organización de la República. Esfuerzo que congregaría a políticos, intelectuales y científicos en tormo de discursos sobre la moder- nización del país. Acompañando estos discursos, ocurrieron un conjunto de cambios sociales, políticos y económicos. Hubo también una inversión importante en la industrialización, y en el desarrollo y renovación científica y pedagógica de Brasil. Entre los años 1930 y 1960 estas iniciativas fueron acentuadas, principalmente, en los gobiernos de Getúlio Vargas y Juscelino Kubitschek con un enfoque en el Sudeste del país.

Este proyecto de modernización fue establecido por la articulación entre la industrialización, el desenvolvimiento científico y la renovación educacional (Mendonça et. al., 2006). Las inversiones en industrialización estaban vinculadas al proceso de urbanización y, consecuentemente al aumento de las poblaciones en las ciudades. Por ejemplo, en 1912, la ciudad de Belo Horizonte (Minas Gerais) poseía cerca de 40.000 habitantes y, en 1950, 350.000. En este escenario, la educación fue tornándose un tema destacado en los discursos e intereses de aquellos políticos, intelectuales y científicos.

La educación fue considerada como uno de los dos principales aspectos de la modernización del Brasil. Desde las décadas de 1920 y 1930 los temas de la universidad y el desarrollo de una ciencia nacional ocupaban las preocupaciones de los intelectuales y científicos que participaban en la educación. Por ejemplo, la Universidade Federal de Minas Gerais (UFMG) fue creada en 1927. A partir de finales de la década de 1950 e inicios de la década de 1960, se profundizó el enfoque sobre la universidad. Este enfoque estaba fuertemente vinculado a la formación de científicos brasileños. Como los cursos de pos-graduación que se fortalecieron a partir del fin de la década de 1960, la graduación debería cumplir las funciones de enseñanza de las habilidades de investigación, incluso desde el inicio de su formación. De esta forma, se acentuó la formación de investigadores brasileños en las universidades en un contexto de carreras de grado.

\section{La Psicología en la Universidad: Carreras de licenciatura en psicología}

Los discursos sobre la universidad y el anhelo de la formación de científicos también impactaron sobre 
la producción de la psicología en Brasil. Ella venía desarrollándose fuertemente en el país desde el inicio del siglo XX, contribuyendo con otras áreas de conocimiento tales como la Pedagogía y la Medicina. Durante las décadas de 1950 y 1960, hubo una sistematización de esfuerzos para que los primeros cursos en psicología comenzaran a funcionar. Las primeras carreras fueron creadas en Rio de Janeiro y Sao Paulo, respectivamente: en la Pontifícia Universidade Católica do Rio de Janeiro (PUC-RJ) en 1953; Universidade de São Paulo (USP) en 1958 y en la Pontifícia Universidade Católica de São Paulo (PUC-SP) en 1962. En la UFMG la carrera de psicología fue creada entre los años de 1962 y 1963.

En este periodo, se estableció, también una legislación para la regulación de la profesión y la formación del psicólogo. En 1962 fue promulgada la Lei $N^{\circ} 4119$ que reglamentaba tales aspectos, y en este mismo año fue publicado el Parecer $N^{\circ} 403$ del Conselho Federal de Educação (CFE). Este Parecer establecía un curriculum mínimo de formación del psicólogo en el país, y destacó la inserción de la psicología experimental en la formación del psicólogo:

... la Psicología General y Experimental, como análisis de los procesos fundamentales del comportamiento (cognición, motivación, y aprendizaje), servirá de apoyo para el entrenamiento de estudiantes en el campo de la experimentación (CFE, 1962).

De esta forma, notamos que la psicología experimental pasó a formar parte del curriculum de las carreras de grado en psicología. Ella estaba presente, principalmente en los laboratorios de psicología experimental, incluyendo dentro de los mismos el análisis experimental del comportamiento.

La década de 1960, marcó la circulación sistemática del análisis experimental del comportamiento en Brasil. Entre 1961 y 1962, Fred Keller estuvo en São Paulo como profesor visitante para ocupar la cátedra de psicología experimental de la Universidade de São Paulo (USP). Keller desenvolvió el uso del laboratorio didáctico que había desarrollado en la Columbia University, EE.UU., con algunos colegas del departamento de psicología (Frick, Schoenfeld \& Keller, 1948). Según sus proponentes, éste tenía por función que los estudiantes realizasen experimentos en lugar de simplemente leer sobre ellos. Su uso didáctico servía de introducción al pensamiento y métodos científicos, como forma de trabajo inicial para hacer una ciencia de laboratorio. Su uso también auxiliaba en la demostración de los hechos sobre el comportamiento, era uno de sus objetos de interés.

Este modelo de laboratorio fue implementado en la USP durante la visita de Keller entre 1961 y 1962. Matos (1998), una de las alumnas brasileñas de Keller relata:

... recuerdo hasta hoy la sensación fuerte y dulce, de que, de ahora en adelante, aprendería no solo de la lectura de los libros, sino haciendo lo que decían los libros, y, finalmente llegando a hacer cosas que ni siquiera estuvieran escritas! (p. 92)

Ella continua diciendo: “... se montaba... un laboratorio de investigación...; y, mas importante todavía, se creaba de la nada un laboratorio de enseñanza, para ser utilizado en el segundo semestre" (p.90, el énfasis es nuestro). Este mismo modelo fue apropiado por otras universidades brasileñas, dentro de ellas la UFMG. (Autor, 2010)

\section{Circulación del Conductismo: El laboratorio didáctico de análisis experimental del comportamiento}

Desde el inicio de la carrera de licenciatura en psicología en la UFMG, circularon temáticas y autores vinculados con el conductismo. Por ejemplo, con fines educativos se realizó una adaptación en 1963 del libro A Análise do Comportamento (The Analysis of Behavior) de James Holland y Burrhus Frederic Skinner. El libro fue adaptado por Ione Scarpelli Pereira, profesora de la UFMG, para el área de psicología del aprendizaje. Sin embargo, la circulación sistematizada del conductismo como análisis experimental del comportamiento ocurrió a partir de 1969. En este año, Carolina Bori fue a la UFMG para dar el curso de psicología social experimental. En encuentros informales con los estudiantes de la UFMG, también ante auxiliares 
y profesores, fueron presentados los principios del análisis experimental del comportamiento y del laboratorio como un recurso didáctico (J. B. Jardim, comunicación personal, 10 de febrero, 2009). Después del contacto con Carolina Bori, parte de los auxiliares y profesores presentes en este curso organizaron un laboratorio didáctico. Instalación que fue llevada a cabo en los primeros años de la década de 1970.

En 1971, un laboratorio precario comenzó a ser utilizado para actividades prácticas de las asignaturas de psicología experimental (S. S. Castanheira, comunicación personal, 5 de febrero, 2009). Maria José Vasconcellos (comunicación personal, 5 de marzo, 2009) afirma: “... el primer lugar donde montamos las cajas fue en el baño.... nosotros no teníamos un laboratorio preparado..." Así, en condiciones precarias, en un baño, fue iniciado el trabajo de laboratorio de análisis experimental del comportamiento en la UFMG. Sônia Castanheira (comunicación personal, 5 de febrero, 2009) nos ofrece más información sobre el funcionamiento de este espacio:

...hacíamos una caja de paloma de cartón..., enseñábamos a cada uno de los alumnos, a hacer su propia caja;... Enseñábamos a ellos como hacer las cajas, y ellos las hacían, ...nosotros estábamos montando un laboratorio experimental de animales en un curso de Psicología, lleno de gente del área de Humanidades ... y confiábamos en estos datos que el alumno obtenía y nos los traía. El alumno realizaba las curvas y todo lo demás, directamente, sobre dichos datos.

Percibimos, a partir de estos relatos, que en esas condiciones precarias de funcionamiento, un laboratorio de análisis experimental del comportamiento fue establecido. Su principal función era contribuir con el aprendizaje de los estudiantes, con la intención de iniciarlos en la producción de datos y realización de curvas de aprendizaje. Elementos comunes de la práctica de psicología experimental en general y del análisis experimental del comportamiento en particular.

Las prácticas llevadas a cabo en este laboratorio inicial de análisis experimental del comportamien- to, con las palomas y las cajas de cartón, estaban basadas en el manual denominado Análise Experimental do Comportamento: Exercícios de laboratório (Kerbauy, 1970). En el prefacio del libro se pueden observar elementos importantes en el desarrollo inicial de ésta práctica de laboratorio en la UFMG:

Un problema de difícil resolución para enseñar psicología experimental en un curso de grado, es mostrar el control experimental de las variables y dar la experiencia personal al estudiante de las técnicas de trabajo en el laboratorio, teniendo poca o ninguna financiación para el equipamiento (Kerbauy, 1970, s.p.).

Según Castanheira (Comunicación personal, 5 de febrero de 2009), este modelo sencillo del baño fue utilizado porque los profesores creían en la base teórica del análisis experimental de la conducta y en su práctica de laboratorio.

En el intervalo entre los años 1971 y 1972, fue realizada una solicitud de equipamiento para un laboratorio de análisis experimental del comportamiento con ratas. De acuerdo con el presupuesto presentado por la Fundação Brasileira para o Desenvolvimento do Ensino de Ciências (FBDE), fueron solicitadas 10 cajas de Skinner para experimentos con ratas. El responsable por este pedido fue João Bosco Jardim y según él ese "laboratorio de análisis experimental tuvo ese papel de enseñanza, cuando se creó: de ser [un] ambiente controlado para las practicas experimentales, que llamábamos ejercicios de laboratorio" (comunicación personal, 10 de febrero, 2009). En este sitio, contaba con una Reglamentación que incluía elementos que hablan de la apropiación didáctica. En este documento, en lo que respecta a los fines del laboratorio, se puede leer:

El Laboratorio de Psicología tiene las siguientes finalidades: a- propiciar las condiciones de entrenamiento para profesores y alumnos en técnicas experimentales; $b$ - propiciar condiciones de enseñanza para alumnos de cursos mantenidos por el Departamento de Psicologia da Faculdade de Filosofia e Ciências Humanas da UFMG (Laboratório de Análise do Comportamento, 1971). 
Notamos que en este documento la enseñanza se pone de relieve, relacionado con el desenvolvimiento de las habilidades y técnicas experimentales, enfocando en alumnos y profesores. Asociando la Reglamentación con las palabras de Jardim (comunicación personal, 10 de febrero, 2009), notamos que el enfoque didáctico estaba en evidencia.

Por lo tanto, podemos observar que el laboratorio era un dispositivo didáctico donde circulaban aspectos relacionados con el análisis del comportamiento. A él le cabía crear las condiciones para la enseñanza de las técnicas experimentales, así como también la demostración de las proposiciones del conductismo skinneriano.

Los profesores del laboratorio del comportamiento crearon dos prácticas experimentales didácticas para complementar el manual de laboratorio utilizado (J. B. Jardim, comunicación personal, 10 de febrero, 2009; S. S. Castanheira, comunicación personal, 5 de febrero, 2009; M. J. E. Vasconcellos, comunicación personal, 5 de marzo, 2009). Las actividades fueron "El comportamiento exploratorio en función del cambio estimulatorio" (Anónimo , s. f.), y "Valor reforzador del sabor dulce no nutritivo" (Anónimo , s.f.). Ambos procedimientos fueron diseñados para ser llevado a cabo con ratones, esto nos sugiere que fueron planeados después de la compra de las cajas de Skinner; es decir, a partir de julio del año 1972. Además, para llevar adelante la primera actividad fue creada por los profesores otra caja experimental. En la descripción del procedimiento, podemos leer:

Será utilizada una caja de observación de madera de $60 \mathrm{~cm}$ de largo, $20 \mathrm{~cm}$ de ancho y $30 \mathrm{~cm}$ alto, con las caras superior y frontal de la malla de alambre y las demás cerradas, con una pequeña escotilla en la parte central de la pared trasera (p.1).

Estos dos ejemplos de prácticas nos ayudan a ver la importancia concedida, al trabajo experimental y a la enseñanza de la psicología. Estos elementos ayudan a comprender las características de la circulación y apropiación del conductismo de la UFMG, a diferencia de aquellas expuestas en la UNSL.
La reconstrucción histórica de este laboratorio, nos ayuda a comprender que los profesores de la UFMG, conseguían con su uso, presentar a los estudiantes los hechos del comportamiento de los organismos conforme a los postulados del análisis experimental del comportamiento. Gradualmente, el laboratorio didáctico se iba configurando como un vector que persuadía a los estudiantes de la UFMG de que los postulados del análisis experimental del comportamiento eran hechos científicos. Estos hechos eran producidos en el laboratorio y por tanto bajo el control del aprendiz de experimentador. Como podemos observar, el laboratorio didáctico de la UFMG nos ayuda a comprender la circulación de prácticas científicas y de una teoría psicológica específica.

\section{Consideraciones Finales}

En ambos procesos se observan elementos diferenciales bien marcados, sin embargo, posiblemente las similitudes fueron las que determinaron que en ambos casos hubiera una recepción de las teorías y prácticas conductistas. Existió en las dos universidades, un fuerte aspecto educacional como antecedente de la constitución de sus carreras de psicología, que también tenía como principal eje la modernización de las técnicas científicas, pero en el caso de la UFMG estaba orientada a la formación de un científico, mientras que en la UNSL a la de un profesional. Parece relevante en ambos casos la asociación de la psicología conductista a la educación y al carácter de una disciplina científica y moderna, efectiva para la generación de conocimientos a través de la experimentación en laboratorios, y práctica para la actuación social de ámbitos como el educativo, el criminológico y el psicoterapéutico.

La distancia geográfica marcó una independencia en la formación ideológica, cultural y conceptual de la psicología de la UNSL, que sumado al antecedente de haber estado asociada su constitución a la carrera de pedagogía permitieron que hubiera una mayor permeabilidad a la hora de receptar la psicología conductista. Esta posición parece ser excepcional en Argentina, donde había una fuerte influencia médica y filosófica con un fuerte énfasis 
en la teoría psicoanalítica en la formación del psicólogo. El aspecto ideológico tiene una influencia marcada en la Argentina y consecuentemente en la UNSL. Si bien por un lado, la asociación de las teorías reflexológicas y conductistas a dos grandes centros imperialistas como la US y EE.UU., fueron motivo de que se las rechazara en los principales centros universitarios donde predominaba una ideología antiimperialista. Por el otro, fue justamente el interés político de los jóvenes de la UNSL, el que permitiera la circulación y apropiación de saberes relacionados al conductismo. Los grupos de la UNSL que promovían el conductismo, lo identificaban como un conocimiento moderno asociado a las ideologías de izquierda, por ende un tipo de psicología más efectiva y práctica a la hora de actuar institucionalmente y socialmente. En este contexto, hubo en una primera instancia una prevalencia mayor de la teoría reflexológicas y conductistas de la que hubo acerca del análisis experimental del comportamiento, que se consolidó recién en 1975 tras los cursos de doctorado impartidos por profesores extranjeros. Finalmente, podemos señalar que la mayoría de las prácticas que se realizaron en la UNSL, fueron en el ámbito profesional a través de las técnicas de la modificación de la conducta.

En el caso de la UFMG, la influencia del carácter científico asociado a una práctica de laboratorio tal como se concebía en los EE.UU., marcó una orientación en la recepción que facilitaría el interés en llevar adelante la conformación tanto de cursos, como de laboratorios, que sirvieran de práctica para la formación de psicólogos actualizados que pudieran contribuir a la modernización de su país. La recepción y apropiación del análisis experimental del comportamiento en la UFMG parece haber ocurrido con un enfoque principal en los aspectos científicos del laboratorio más que en las convicciones ideológicas. En este contexto, las fuentes sugieren que hubo una prevalencia del uso didáctico del laboratorio, contribuyendo a la formación científica del psicólogo. Las referencias bibliográficas fueron, sobretodo, manuales de laboratorio para auxiliar en las prácticas didácticas. La creencia de los profesores en la teoría y experimentación del análisis del comportamiento, así como, la importancia de la enseñanza de una psicología científica, fomentaron su desarrollo con la creación de los laboratorios. Este enfoque parece tener relación con la creencia en el perfeccionamiento de un científico que contribuyera al desarrollo de la ciencia brasileña.

Para finalizar, debemos mencionar las limitaciones de nuestro trabajo. Como estudiamos los casos de dos universidades, la UNSL y la UFMG, no podemos extrapolar nuestros datos para todas las universidades del país en el mismo periodo. Nuevos estudios deberían ser llevados a cabo en otras instituciones para poder realizar generalizaciones para cada uno de estos países. A su vez, consideramos que futuros trabajos deben considerar otros aspectos del contexto de ambos países durante las décadas de 1960 y 1970. Tampoco podemos realizar generalizaciones sobre otros países de América Latina, a pesar de que haya un conjunto de similares características en sus contextos de aquel periodo. Por ende, para profundizar la relación entre los países latinoamericanos en lo que atañe a la circulación y apropiación del conductismo se espera realizar estudios más amplios. Sin embargo, nuestro estudio señala características de la recepción del conductismo que nos auxilian en la comprensión de los caminos iniciales de esta teoría en los diferentes lugares de América Latina.

\section{Referencias}

Anônimo (s.d.). Comportamento exploratório em função da mudança estimulatória. Instruções para Realização do Experimento em que constam Introdução, Método (subdividido em sujeito e equipamento) e Procedimento. Fotocopiado. 3f.

Anônimo (s.d.). Valor Reforçador do Gosto Doce NãoNutritivo. Instruções para Realização do Experimento em que apenas Seção de Procedimento Experimental. Fotocopiado. 1f.

Bloch, M. (1999). Historia e historiadores. Madrid: Akal. Buchbinder, P. (2010). Los sistemas universitarios de Argentina y Brasil: una perspectiva histórica y comparada de su evolución desde mediados del siglo XX. En P. Buchbinder, J. Califa y M. Millán (Eds), Apuntes sobre la formación del movimiento 
estudiantil argentino (1943-1973) (pp. 9-30). Buenos Aires: Final Abierto.

Calabresi, Corina \& Polanco, Fernando (2008). Un precedente de psicología conductual en la Universidad Nacional de San Luis (Argentina). Memorandum. Memória e História em Psicología, 15, 52-60.

Capelato, M. H. R. (1998). Multidões em Cena: Propaganda política no varguismo e no peronismo. São Paulo: Papirus e FAPESP.

Danziger, K. (2006). Universalism and indigenization in the history of modern psychology. En A. C. Brock (Ed.), Internationalizing the history of psychology (pp. 208-225). New York: New York University Press.

Frick, F., Schoenfeld, W., \& Keller, F. (1948). Apparatus Designed for Introductory Psychology of Columbia College. The American Journal of Psychology, 61(3), $409-414$.

Hurtado, D. (2010). La ciencia Argentina. Un proyecto inconcluso: 1930-2000. Buenos Aires: Edhasa.

Kerbauy, R. R. (1970). Análise Experimental do Comportamento: Exercícios de laboratório. São Paulo: sem editora.

Klappenbach, H. (2006). Periodización de la psicología en Argentina. Revista de historia de la Psicología, 27 (1), 109-164.

Laboratório de Análise do Comportamento. (1971). Regulamento do Laboratório de Psicologia. Belo Horizonte: Universidade Federal de Minas Gerais.
Matos, M. A. (1998). Contingências para a Análise Comportamental no Brasil. Psicologia USP, 9(1), $89-100$.

Mendonça, A. W. P. C.; Xavier, L. N.; Breglia, V. L. A.; Chaves, M. W.; Oliveira, M. T. C.; Lima, C. N.; \& Santos, P. S. M. B. (2006). Pragmatismo e desenvolvimentismo na pensamento educacional brasileiro dos anos de 1950/1960. Revista Brasileira de Educação, 11(31), 96-113.

Miranda, R. L. \& Cirino, S. D. (2010). Os Primeiros Anos dos Laboratórios de Análise do Comportamento no Brasil. Psychologia Latina, 1(1), 79-87.

Obregón, D. (2000). Culturas científicos y saberes locales: asimilación, hibridación, resistencia. Bogotá: Universidad Nacional de Colombia.

Plotkin, M. (2003). Freud en las Pampas. Buenos Aires: Sudamericana.

Puiggrós, A. (2003). Quépaso en la educación Argentina. Buenos Aires: Galerna.

Rodríguez-Kauth, A. (1973). Estado actual de la situación del Psicólogo en San Luis. Revista de Psicología, 6, 105-115.

Thénon, J. (1963). Prólogo. En Wells, H., Iván P. Pavlov hacia una Psicología y Psiquiatría Científicas (pp. 9-16). Buenos Aires: Platino.

Universidad Nacional de San Luis (1975). Plan docente. San Luis: UNSL. Mimeo. 
